\title{
Targeted Inhibition of FGF19/FGFR Cascade Improves Anti-Tumor Immunity and Response Rate in Hepatocellular Carcinoma
}

\section{David Wai Meng Tai}

National Cancer Centre Singapore

Thi Bich Uyen Le

National Cancer Centre Singapore

\section{Aldo Prawira}

National Cancer Centre Singapore https://orcid.org/0000-0003-4414-6928

\section{Rebecca Zhi Wen Ho}

National Cancer Centre Singapore

Hung Huynh ( $\nabla$ cmrhth@nccs.com.sg )

National Cancer Centre Singapore https://orcid.org/0000-0001-7847-3981

\section{Research Article}

Keywords: Preclinical study, liver cancer, PDX model, fibroblast growth factor signaling pathway, growth inhibition, drug resistance, metastasis, immune response, overall survival, vascular normalization, tumor hypoxia.

Posted Date: April 5th, 2021

DOl: https://doi.org/10.21203/rs.3.rs-343037/v1

License: @ (i) This work is licensed under a Creative Commons Attribution 4.0 International License. Read Full License

Version of Record: A version of this preprint was published at Hepatology International on July 31st, 2021. See the published version at https://doi.org/10.1007/s12072-021-10212-8. 


\section{Abstract \\ Background}

Hepatocellular carcinoma (HCC) is the most common liver cancer globally, claiming nearly 1 million lives each year. Overexpression of fibroblast growth factor (FGF) receptors (FGFRs) signaling cascade has been shown to contribute to tumorigenesis, metastasis, and poor prognosis in HCC. Therefore, targeted inhibition of the FGF/FGFR cascade may represent a new treatment strategy for HCC patients.

\section{Methods}

HCC patient-derived xenograft (PDX) models were implanted into either severe combined immunodeficient (SCID) or CD34 + hu-NSG (humanized) mice and subsequently treated with vehicle, infigratinib (FGFR1-3 inhibitor), FGF401 (FGFR4 inhibitor), or the combination of infigratinib and FGF401. Tumor progressions, overall survival of mice, lung metastasis, and drug resistance were monitored, and samples collected at the end of the treatment cycle were subjected to Western blot analyses and immunohistochemistry.

\section{Results}

HCC PDX models expressing high levels of FGF19/FGFR4 or FGFR2/3 showed favorable initial treatment response to FGF401 and infigratinib, respectively. However, progressive disease due to acquired resistance was observed. Combination infigratinib/FGF401 augmented the antitumor activity, response rate, and overall survival of mice. This combination significantly increased the infiltration of B-cells, macrophages, CD8 + T-cells, and CD4 + T-cells associated with granzyme-B mediated apoptosis, delayed onset of resistance, and inhibited metastasis by potently inhibiting several critical signaling pathways involved in proliferation and metastasis.

\section{Conclusions}

Our findings suggest that HCC patients with high FGFR2/3 or FGF19/FGFR4 expressing tumors might benefit from a combination infigratinib/FGF401, thus supporting its evaluation in clinical trials.

\section{Introduction}

Hepatocellular carcinoma (HCC) is amongst the five most common cancers globally, with nearly 1 million new cases diagnosed annually [1]. Currently, sorafenib, lenvatinib, and combination of atezolizumab and bevacizumab are approved by the FDA as first-line treatment options. In addition, regorafenib and cabozantinib are indicated to be second-line options (reviewed in [2]). A randomized phase III study of programmed cell dealth protein 1 (PD-1) inhibitor nivolumab (NCT02576509) failed to achieve its primary 
endpoint of overall survival (OS). Given the increasing incidence of the disease around the world [1], newly effective treatments for advanced HCC are needed.

Overexpression of fibroblast growth factor (FGF) receptor (FGFR)-2/3 has been associated with tumorigenesis, metastasis, propensity for distant recurrence, poorly differentiated tumor, and poor prognosis in advanced HCC [3, 4]. Additionally, high levels of FGFs have been shown to increase vascular endothelial growth factor (VEGF)-mediated HCC development and angiogenesis [5] potentially leading to resistance to VEGF/VEGF receptor-targeted agents [6]. FGF19 amplification or overexpression has been detected in approximately $14 \%$ and $50 \%$ of HCC patients, respectively [7]. FGFR4 and FGF19 expression correlates with poorer prognosis, recurrence, and shorter OS in HCC patients [7]. In transgenic mice, overexpression of FGF19 produces liver tumors that are sensitive to antibodies against FGFR4 or FGF19 $[8,9]$. Furthermore, FGFR4 knockout prevented transgenic mice with exogenous FGF19 expression from developing tumors. These studies suggest the involvement of FGF19/FGFR4 and FGFR2/3 in the pathogenesis of HCC and provide a strong rationale for combined targeting FGF19/FGFR-4 and FGFR2/3 in HCC patients with FGFR-dependent tumors $[9,10]$.

Infigratinib, a pan-FGFR inhibitor, has been reported to inhibit bladder cancer xenografts and basic FGF (bFGF)-stimulated angiogenesis without impairing VEGF-induced blood vessel formation [11]. Infigratinib potently suppresses the growth of HCC xenografts by inhibiting FGFR signaling and its downstream targets, therefore reducing cell proliferation, angiogenic rescue program, intratumoral hypoxia, and metastasis [12]. It also acts in synergy with vinorelbine to promote apoptosis, suppress tumor growth, and improve OS of mice bearing FGFR-dependent HCCs [12].

FGF401 is a selective FGFR4 inhibitor that has shown potent antitumor activity in patient derived xenograft (PDX) models that are positive for FGF19 [13, 14]. FGF401 also reduced tumor hypoxia, induced blood vessel normalization, prolong the OS of mice bearing in a FGF19-dependent manner [14]. An ongoing Phase I/II study of FGF401 in patients with HCC or solid tumors with positive FGFR4 and klotho $\beta$ expression (NCT02325739) demonstrated promising clinical activity and a favorable safety profile [15].

The goals of the present study were to decipher the underlying mechanisms of abrogating FGFR2/3 and FGF19/FGFR4 in HCC PDX, and their mechanisms of secondary resistance. We demonstrated that combination infigratinib/FGF401 provided a robust antitumor activity in FGFR2/3 or FGF19/FGFR4 highexpressing HCC models by potently inhibiting signaling pathways that are involved in promoting cell proliferation, metastasis, survival and drug resistance. This combination also significantly increased the infiltration of B-cells, macrophages, CD8 + T-cells, and CD4 + T-cells associated with granzyme-B mediated apoptosis. Our results provide the possibility of a biomarker-directed therapeutic approach aimed at complete functional abrogation of FGF19/FGFR2-4 cascade in the management of HCC.

\section{Materials And Methods}


The details for drug preparation and administration, and statistical analyses were described in Supplementary Materials and Methods.

Xenograft models. All animals received humane care according to criteria outlined in "Guide for the Care and Use of Laboratory Animals" [16]. Mice were provided with sterilized food and water ad libitum, and housed in negative pressure isolators set at $23^{\circ} \mathrm{C}$ and $43 \%$ humidity with 12 hour light/dark cycles.

Previously established patient-derived xenograft (PDX) lines were subcutaneously implanted in male C.B17 SCID mice (InVivos Pte. Ltd., Singapore) aged 9-10 weeks, weighing 23-25 grams, as previously described [12]. The characteristics of the 9 PDX models used in this study are shown in Supplementary Table 1. For dose-titration experiments, mice bearing high FGFR2/3-expressing HCC01-0909 and high FGF19-expressing HCC09-0913 ( $n=10$ mice per group) were administered $200 \mu \mathrm{l}$ vehicle, $15 \mathrm{mg} / \mathrm{kg}$ infigratinib, $30 \mathrm{mg} / \mathrm{kg}$ FGF401, or the combination of both in decreasing concentration (i.e. 15:30, 10:20, $7.5: 15,5: 10 \mathrm{mg} / \mathrm{kg}$ infigratinib:FGF401) for 15 and 9 days, respectively.

\section{The efficacy of infigratinib/FGF401 in ectopic HCC models.}

Mice bearing tumors ( $n=8-10$ per group) were orally treated as follows: 1) $200 \mu$ l vehicle (control); 2) 15 $\mathrm{mg} / \mathrm{kg}$ infigratinib; 3) $30 \mathrm{mg} / \mathrm{kg}$ FGF401; 4) combination infigratinib and FGF401; or 5) $10 \mathrm{mg} / \mathrm{kg}$ sorafenib. Treatment was initiated when tumors reached approximately $170-250 \mathrm{~mm}^{3}$. Tumor growth was monitored, and volume calculated as previously described [12]. At the end of the study, mice were euthanized by $\mathrm{CO}_{2}$ asphyxiation $2 \mathrm{~h}$ after the last dose of treatment. Body and tumor weights were recorded, and tumors harvested for subsequent analyses.

The efficacy of infigratinib, FGF401, and combination infigratinib/FGF401 was determined by the T/C ratio, where $\mathrm{T}$ and $\mathrm{C}$ are the median weights of drug-treated and vehicle-treated tumors, respectively, at the end of treatment. $\mathrm{T} / \mathrm{C}$ ratios less than 0.42 were considered active [12].

\section{The efficacy of infigratinib/FGF401 in orthotopic HCC models.}

HCC09-0913 and HCC01-0909 orthotopic models were established as previously described [17]. Mice were randomized ( $n=10$ per group) and treatment initiated when tumors reached approximately $100-$ $150 \mathrm{~mm}^{3}$. For survival, metastasis, and invasion experiments, mice bearing tumors were treated with infigratinib, FGF401, or combination infigratinib/FGF401 as described above for 28 days. Body weight, ascites formation, and OS were monitored daily. Mice were sacrificed when they became moribund, and the extent of macroscopic lung metastasis and sizes of the primary orthotopic tumors were documented.

\section{The efficacy of infigratinib/FGF401 in humanized HCC01-0909 model.}

HCC01-0909 tumors were subcutaneously implanted in female CD34 + hu-NSG humanized mice as described in Supplementary Materials and Methods. Mice were subsequently randomized into 4 groups ( $n=5$ mice per group) and treated as follows: 1) $200 \mu \mathrm{l}$ vehicle (control); 2) $15 \mathrm{mg} / \mathrm{kg}$ infigratinib; 3) 30 $\mathrm{mg} / \mathrm{kg}$ FGF401; or 4) combination infigratinib and FGF401. Treatment was initiated when tumors reached 
approximately $170-250 \mathrm{~mm}^{3}$. Tumor growth was monitored and volume calculated as described previously [12].

\section{Results}

\section{Infigratinib and FGF401 synergistically inhibited tumor growth in HCC PDX models}

We first compared the antitumor activity of combination infigratinib/FGF401 with infigratinib, FGF401, and sorafenib in HCC models that express either FGF19, FGFR2/3/4, or both (Supplementary Table 1). Both infigratinib and FGF401 had similar antitumor efficacy in the HCC09-0913 model (high FGF19 and FGFR2/3/4; Fig. 1A-C). Infigratinib showed better antitumor activity than FGF401 or sorafenib in the HCC01-0909 model (High FGFR2/3/4 but very low FGF19; Fig. 1D-F, $p<0.05$ ). Sorafenib inhibited tumor growth by approximately 40\% in HCC09-0913 and 50\% in HCC01-0909 models (Fig. $1, p<0.05$ ). In contrast, combination infigratinib/FGF401 exerted nearly complete growth inhibition and showed superior antitumor activity than FGF401 or infigratinib alone in both models $(p<0.05)$. Supplementary Table 2 shows the T/C ratios for nine HCC models treated with infigratinib, FGF401, or combination infigratinib/FGF401. For the eight FGFR-dependent models, the response rates for infigratinib, FGF401 and infigratinib/FGF401 were 50\% (4/8 with T/C < 0.42), 50\% (4/8 with T/C < 0.42$)$ and 100\% (8/8 with $\mathrm{T} / \mathrm{C} \leq 20 \%$ ), respectively. Although the sorafenib-resistant HCC06-0606Sora87 model was modestly sensitive to infigratinib and completely resistant to FGF401, its growth was significantly suppressed by combination infigratinib/FGF401 ( $p<0.001$, Supplementary Table 2).

FGF401 significantly inhibited the growth of high FGF19/FGFR4-expressing HCC models (HCC09-0913, HCC13-0212, HCC26-1004, and HCC29-1104) but had no activity in high FGFR2/3-expressing but undetectable FGF19 models (HCC26-0808A, HCC06-0606, HCC06-0606Sor87) (Supplementary Table 2, Supplementary Data 1). Conversely, infigratinib significantly inhibited the growth of high FGFR2/3 but undetectable FGF19 expressing models. HCC01-0909 model was sensitive to infigratinib and modestly to FGF401 (Supplementary Table 2; Fig. 1D-F). FGF401 synergistically acted with infigratinib to enhance antitumor efficacy in all 8 HCC models tested (Supplementary Table 2). In contrast, infigratinib, FGF401, and combination infigratinib/FGF401 exhibited no significant antitumor activity in HCC10-0505 model, which expressed undetectable levels of FGF19, FGFR1/2 and low levels of FGFR3 (Supplementary Table 2; Supplementary Data 1).

We next sought to determine if lower doses of both drugs could achieve antitumor efficacy similar to that of standard doses. Treatment of mice bearing HCC09-0913 tumors with 15:30, 10:20, and 7.5:15 mg/kg infigratinib:FGF401 for 9 days led to approximately $97.2 \%, 93.2 \%$, and $88.5 \%$ reductions in tumor burden, respectively (Fig. 2A-C, $p<0.0001$ ). Similarly, treatment of mice bearing HCC01-0909 xenografts with $15: 30,10: 20,7.5: 15$, and 5:10 mg/kg infigratinib:FGF401 for 15 days led to approximately $98.0 \%, 98.3 \%$, $97.1 \%$, and $96 \%$ reductions in tumor burden, respectively (Fig. 2D-F, $p<0.0001$ ). In these two models, 10 $\mathrm{mg} / \mathrm{kg}$ infigratinib and $20 \mathrm{mg} / \mathrm{kg}$ FGF401 yielded efficacy similar to that as the standard dose of 15 
$\mathrm{mg} / \mathrm{kg}$ infigratinib and $30 \mathrm{mg} / \mathrm{kg}$ FGF401, suggesting that dose reduction is possible without compromising the efficacy. Throughout the course of treatment, no significant body weight loss or other signs of toxicity were observed in mice from treatment groups compared with those from the vehicle group, suggesting that the treatments are well-tolerated (Supplementary Data 2A). Moreover, combination infigratinib/FGF401 resulted in the increase in white blood cells, lymphocytes, monocytes, platelets, serum alanine aminotransferase (ALT), alkaline phosphatase (ALP), and aspartate aminotransferase (AST), and a significant decrease in serum creatinine (Supplementary Data $2 \mathrm{~B}, p<0.01$ ).

\section{Combination infigratinib/FGF401 induced cell cycle arrest and apoptosis in HCC cells in vitro}

Infigratinib and FGF401 significantly induced G1 cell cycle arrest (Supplementary Data 1A). Cells treated with combination infigratinib/FGF401 showed a higher proportion of sub-G1 phase in a dose-dependent manner, compared with those treated with infigratinib or FGF401 alone, suggesting that the combination is more potent than a single agent in inducing apoptosis.

\section{Combination infigratinib/FGF401 induced apoptosis, inhibited cell proliferation, switched off the angiogenic rescue program and reduced tumor hypoxia via blood vessel normalization}

In high FGF19 expressing HCC09-0913 model, FGF401 was more potent than infigratinib in inhibiting cell proliferation but had similar potency to induce apoptosis. FGF401 was more potent than infigratinib to induce apoptosis but had similar potency to inhibit cell proliferation in HCC13-0212 (Supplementary Data 3A). In contrast, infigratinib, but not FGF401, significantly inhibited cell proliferation in high FGFR2/3 expressing HCC01-0909 model (Fig. 3A, $p<0.01$ ). Both Infigratinib and FGF401 had minimal or no effect on apoptosis in HCC01-0909 model (Fig. 3B). Neither FGF401 nor infigratinib had any significant effect on proliferation or apoptosis of low FGFR2/3 HCC10-0505 tumors (Supplementary Data 3B). In high FGFR2/3- or FGF19-expressing tumors, the infigratinib/FGF401-treated group showed a lower number of

p-Histone 3-positive cells and more cleaved PARP-positive cells than treatment with infigratinib or FGF401 alone (Fig. 3A-B, Supplementary Data $3 A-B, p<0.001$ ), suggesting a combination effect in promoting apoptosis and inhibition of cell proliferation.

Blood vessels in the infigratinib- and to a lesser extent, FGF401-treated HCC09-913 and HCC01-0909 tumors were slim, resembling capillary-like vessels (Fig. 3A-B). Furthermore, the majority of capillary-like blood vessels induced by infigratinib and combination infigratinib/FGF401 were positive for lectin, suggesting productive blood vessels. Hypoxyprobe staining was negative across large sections of these tumors, indicating that the regions were well-oxygenated. In HCC09-0913, combination FGF401/infigratinib did not induce any significant alteration in total blood vessels, number of lectinpositive blood vessels, and tumor hypoxia compared with infigratinib or FGF401 monotherapy (Fig. 3, Supplementary Data 4). In HCC01-0909, significant increase in total blood vessels and number of lectinpositive blood vessels were observed (Fig. 3B, Supplementary Data 4, $p<0.01$ ). Neither FGF401 nor infigratinib had any significant effect on total blood vessels of low FGFR2/3 HCC10-0505 tumors (Supplementary Data 3B). 
We next analyzed the mRNA levels of proangiogenic factors in vehicle- and drug-treated tumors using qRT-PCR. In the high FGFR2/3 HCC21-0208 model, infigratinib- and FGF401-treated tumors had two-fold lower levels of VEGF and HIF-1a than vehicle-treated tumors (Supplementary Data 3C, $p<0.05$ ). bFGF expression was undetected in infigratinib-, FGF401-, and combination infigratinib/FGF401-treated samples. Combination infigratinib/FGF401 further decreased the levels of VEGF, CYR61, PDGF-AA, and HIF-1a mRNAs. Infigratinib-treated high FGFR2/3-expressing HCC01-0909 tumors showed a four-fold lower level of VEGF mRNAs and a two-fold lower level of CYR61 mRNAs compared to the vehicle-treated tumors in the model (Supplementary Data 3D, $p<0.05$ ). Further reduction of VEGF, CYR61, and PDGF-AA mRNA levels were observed with combination infigratinib/FGF401. Similar data were obtained when mice bearing high FGF19-expressing HCC13-0212 tumors were treated with combination infigratinib/FGF401 (Supplementary Data 3E). There were no significant changes in the expression of proangiogenic factors in low FGFR-expressing HCC10-0505 tumors treated with infigratinib, FGF401, or combination infigratinib/FGF401 (Supplementary Data 3F).

\section{Combination Infigratinib/fgf401 Inhibited Fgfr Signaling And Its Downstream Targets}

Infigratinib, but not FGF401, inhibited p-FRS2 $a$ and p-ERK1/2 in HCC01-0909 and HCC06-0606 (high FGFR2/3 and undetectable FGF19). Both FGF401 and infigratinib potently reduced p-FRS2 $a$ and pERK1/2 in HCC09-0913 (high FGF19 and FGFR2/3 expression). In the three models analyzed, treatment with combination infigratinib/FGF401 further reduced the levels of FGFR2/3, p-FRS2a, pp70S6K/4EBP1/S6R, p-ERK1/2, p-Cdk2, p-Cdc2, CDC25C, p-Rb, p-Cdc2, c-Myc, cyclin D1, and survivin compared with infigratinib or FGF401 treatment alone. Significant upregulation of p27, dephosphorylated form of Bim (fast-migrated form) and cleaved caspase 3 were detected in infigratinib/FGF401-treated tumors ( $p<0.05$; Fig. 4). However, changes in p-ERK1/2, p-AKT, survivin, $\mathrm{p}$-Cdk2 and cyclin D1 were not detected in the infigratinib/FGF401-treated HCC10-0505 tumors, which expressed low FGFRs and nullFGF19 (Supplementary Data 5).

\section{Combination infigratinib/FGF401 inhibited tumor growth and lung metastasis, and prolonged the survival of mice bearing orthotopic tumors}

As shown in Fig. 5A, infigratinib but not FGF401 significantly suppressed the growth of high FGFR2/3expressing HCC01-0909 tumors $(p<0.01)$. However, combination infigratinib/FGF401 showed further inhibition of tumor growth compared to infigratinib or FGF401 alone $(p<0.01)$. In this model, lung metastasis was detected in $90 \%(9 / 10), 20 \%(2 / 10), 80 \%(8 / 10)$, and $0 \%(0 / 10)$ of vehicle-, infigratinib-, FGF401-, and infigratinib/FGF401-treated mice, respectively. The survival of mice bearing HCC01-0909 tumors treated with combination infigratinib/FGF401 was significantly longer than that of mice treated with single agents (Fig. 5B; $p<0.001$, log-rank test). For the high FGF19- and FGFR2/3-expressing HCC090913 model, more than $60 \%$ of the mice in the combination group were still alive on day 250 (Fig. 5B; $p<$ 0.001 , log-rank test). Thus, combination infigratinib/FGF401 inhibited lung metastasis and prolonged the survival of mice bearing FGF19- or FGFR2/3-dependent tumors. 
Combination infigratinib/FGF401 induced immune cell infiltration and apoptosis contributing to an antitumor effect

We next investigated whether inhibition of the FGF19/FGFR signaling pathway improves intratumoral immune cell infiltration. Compared with infigratinib or FGF401 alone, combination infigratinib/FGF401 caused insignificant changes on body weight (Fig. 6A) but significantly reduced tumor growth (Fig. 6B) and tumor weight (Fig. 6C). The tumor regression in Infigratinib/FGF401 was associated with significant increase in the infiltration of CD8 + T-cells, CD4 + T-cells, CD11c + dendritic cells, B-cells, and macrophages concomitant with granzyme-B mediated apoptosis (Supplementary Data 7A-B, $p<0.01$ ). In contrast, infigratinib or FGF401 monotherapy caused an insignificant increase in immune cell infiltration (Supplementary Data 7B).

\section{Discussion}

In the present study, HCC PDX models expressing different levels of FGFRs and FGF19 were used to investigate the role of FGFR signaling in growth, metastasis, and overall survival because PDX models can better capture the molecular diversity and cellular heterogeneity observed in patient tumors. We demonstrated that combination infigratinib/FGF401 abrogated the activity of both FGFR2/3 and FGF19/FGFR4 signaling, thus improving and prolonging response and reducing metastasis, resulting in improved OS. Combination infigratinib/FGF401 (1 infigratinib:4 FGF401) exerted a potent antitumor activity in HCC models that are FGFR2/3- or FGF19-dependent by inhibiting cell proliferation and inducing apoptosis. In orthotopic models, combination infigratinib/FGF401 showed significant inhibition of tumor growth and lung metastasis, and prolonged survival in mice. This combination lowered the effective dose of each agent by $33.3 \%$ but still retained enhanced activity, thus reducing the likelihood of drug toxicity and selection pressure for development of drug resistant clones.

The antitumor effects of combination infigratinib/FGF401 in high FGFR2/3- or FGF19-expressing HCC models were the result of dual mechanisms of action: inhibition of tumor cells and alteration of tumor microenvironment. The higher antitumor activity observed in infigratinib/FGF401-treated mice is probably due to inhibition of FGFR, Raf/MEK/ERK, and PI3K/Akt/mTOR pathways, which are implicated in HCC development, metastasis, and resistance to VEGFR modulating agents $[6,18,19]$. Combination infigratinib/FGF401 significantly increased apoptosis without impairing blood vessel normalization induced by infigratinib or FGF401 alone. Importantly, we observed the possibility of immune-mediated cell killing in combination infigratinib/FGF401, but not in infigratinib or FGF401 single agents in humanized mouse model.

Immunotherapy relies on the accumulation and activity of immune effector cells within the tumor microenvironment. Previous research indicated that immune responses and vascular normalization are reciprocally regulated [20]. We observed that infigratinib/FGF401 induces the favorable microenvironment required to achieve an effective and sustained immune response. This combination significantly increases the infiltration of the immune effector cells into the tumors, presumably due to vessel 
normalization and reduction in tumor hypoxia. Combination infigratinib/FGF401 resulted in the conversion of the intrinsically immunosuppressive microenvironment to an immunosupportive one, providing the basis for the addition of immunotherapy [20-22]. This allowed greater intra-tumoral CD4+ and CD8 + T-cells, CD11c + dendritic cells, CD4-positive granzyme B, and CD68 + macrophage infiltration, resulting in superior tumor regression compared with monotherapy (Fig. 6B-C and 7). Our data are supported by earlier reports showing that infiltration of the aforementioned immune cells correlates with better prognosis [23]. Consistently, patients with higher tumor infiltrating lymphocytes, particularly CD8 + T-cells, showed better clinical response to immunotherapies compared to patients with lower infiltrates [24-26].

Furthermore, combination infigratinib/FGF401 caused G1 cell cycle arrest and apoptosis by reducing levels of p-p70S6K/4EBP1/S6R, p-Cdk2, p-Cdc2, CDC25C, p-Cdc2, Cyclin D1, c-Myc and survivin compared with infigratinib or FGF401 treatment with a concomitant upregulation of $p 27$, dephosphorylated form of Bim (fast-migrated form) and cleaved caspase 3. We showed that metastatic potential of the tumors was diminished or lost in combination infigratinib/FGF401. Although the exact mechanism responsible for this remains undetermined, inhibition of the ERK and p70S6K pathways could partly explain this finding. Notably, we demonstrated that the dose of infigratinib and FGF401 could be reduced by $33 \%$ without compromising its efficacy (Fig. 2). It remains to be determined if combination infigratinib/FGF401 results in cholestasis in clinical settings.

We identified a drug combination that potently inhibits tumor growth and metastasis and significantly improves OS. Infigratinib or FGF401 monotherapies led to regression of FGFR2/3- or FGF19-driven tumors, respectively. Nevertheless, the emergence of acquired resistance to single-agent FGFR treatment limits the long-term benefit. Based on this observation, we anticipate that patients with FGFR2/3overexpressing HCC could derive a benefit when treated with infigratinib but not FGF401, whereas patients with high FGF19/FGFR4-expressing HCC could derive a benefit when treated with FGF401 but not infigratinib. Combination infigratinib and FGF401 blocked several signaling circuits, thus overcoming resistance with single agent therapy. It also converts the immunosuppressive microenvironment to an immunosupportive one, allowing greater intra-tumoral immune cell infiltration, resulting in superior tumor regression compared with monotherapy. Taken together, our data provide the rationale to evaluate combination infigratinib/FGF401 in HCC patients with high FGFR2/3 ( 17\%) [12] or FGF19 ( 12\%) expression [14].

\section{List Of Abbreviations}

\section{bFGF}

Basic fibroblast growth factor

CYP3A4

Cytochrome P450 3A4

CYR61

Cysteine-rich angiogenic inducer 61 
EZH2

Enhancer of zeste homolog 2

FGF

Fibroblast growth factor

FGFR

Fibroblast growth factor receptor

HCC

Hepatocellular carcinoma

HIF-1a

Hypoxia-inducible factor 1-alpha

OS

Overall survival

PDGF-AA

Platelet-derived growth factor with two A subunits

PDX

Patient derived xenograft

SCID

Severe combined immunodeficient

VEGF

Vascular endothelial growth factor

VEGFR

Vascular endothelial growth factor receptor

\section{Declarations}

\section{Funding}

This work was supported by Singapore National Medical Research Council - Ministry of Health Industry Alignment Fund Category 2 Grant NMRC/MOHIAFCAT2/006/2016 (H.H.), Singapore National Medical Research Council - Ministry of Health Industry Alignment Fund Category 1 Grant NMRC/MOHIAFCat1/0026/2015 and NMRC/MOHIAFCat1/0009/2014 (H.H.), the RIE2020 NCIS Centre Grant, grant number CGAug16M005 (H. H.), and Singapore National Research Foundation NRF-CRP172017-05 (H.H.).

\section{Conflicts of interest}

Not applicable.

\section{Ethics approval}

This study received ethics board approval at SingHealth and National Cancer Centre Singapore (IACUC \#2018/SHS/1401 and \#2012/SHS/731). This study involves the use of human biological material 
(patient-derived xenografts) that is not individually identifiable.

\section{Consent to participate}

Consent to collect tumor samples from patients were obtained by the SingHealth Tissue Repository prior to tissue manipulation to generate xenografts.

\section{Consent for publication}

Not applicable.

\section{Availability of data and materials}

The datasets used and/or analyzed during the current study are available from the corresponding author on reasonable request.

\section{Code availability}

Not applicable.

\section{Authors' contributions}

Conception and study design: $\mathrm{HH}$ and DTWM.

Development of methodology: $\mathrm{HH}$.

Acquisition of data: TBUL, AP, RHZW.

Analysis and interpretation of data: DTWM and $\mathrm{HH}$.

Writing, review, and/or revision of manuscript: DTWM, TBUL, AP, and $\mathrm{HH}$.

Study supervision: $\mathrm{HH}$.

\section{References}

1. Siegel RL, Miller KD, Jemal A. Cancer Statistics, 2017. CA Cancer J Clin. United States; 2017;67:7-30.

2. Gordan JD, Kennedy EB, Abou-Alfa GK, Beg MS, Brower ST, Gade TP, et al. Systemic Therapy for Advanced Hepatocellular Carcinoma: ASCO Guideline. J Clin Oncol Off J Am Soc Clin Oncol. United States; 2020;38:4317-45.

3. Harimoto N, Taguchi K, Shirabe K, Adachi E, Sakaguchi Y, Toh Y, et al. The significance of fibroblast growth factor receptor 2 expression in differentiation of hepatocellular carcinoma. Oncology. Switzerland; 2010;78:361-8.

4. Paur J, Nika L, Maier C, Moscu-Gregor A, Kostka J, Huber D, et al. Fibroblast growth factor receptor 3 isoforms: Novel therapeutic targets for hepatocellular carcinoma? Hepatology. United States; 
2015;62:1767-78.

5. Kano MR, Morishita Y, Iwata C, Iwasaka S, Watabe T, Ouchi Y, et al. VEGF-A and FGF-2 synergistically promote neoangiogenesis through enhancement of endogenous PDGF-B-PDGFRbeta signaling. $J$ Cell Sci. England; 2005;118:3759-68.

6. Bergers G, Hanahan D. Modes of resistance to anti-angiogenic therapy. Nat Rev Cancer. 2008;8:592603.

7. Hyeon J, Ahn S, Lee JJ, Song DH, Park C-K. Expression of fibroblast growth factor 19 is associated with recurrence and poor prognosis of hepatocellular carcinoma. Dig Dis Sci. United States; 2013;58:1916-22.

8. Zhou M, Wang X, Phung V, Lindhout DA, Mondal K, Hsu J-Y, et al. Separating Tumorigenicity from Bile Acid Regulatory Activity for Endocrine Hormone FGF19. Cancer Res. United States; 2014;74:3306-16.

9. French DM, Lin BC, Wang M, Adams C, Shek T, Hotzel K, et al. Targeting FGFR4 inhibits hepatocellular carcinoma in preclinical mouse models. PLoS One. United States; 2012;7:e36713.

10. Sawey ET, Chanrion M, Cai C, Wu G, Zhang J, Zender L, et al. Identification of a therapeutic strategy targeting amplified FGF19 in liver cancer by Oncogenomic screening. Cancer Cell. United States; 2011;19:347-58.

11. Guagnano V, Furet P, Spanka C, Bordas V, Le Douget M, Stamm C, et al. Discovery of 3-(2,6-dichloro3,5-dimethoxy-phenyl)-1-\{6-[4-(4-ethyl-piperazin-1-yl)-phenylamino]-pyrimidin-4-yl\}-1-methyl-urea (NVP-BGJ398), a potent and selective inhibitor of the fibroblast growth factor receptor family of receptor tyrosine kinase. J Med Chem. United States; 2011;54:7066-83.

12. Huynh H, Lee LY, Goh KY, Ong R, Hao H-X, Huang A, et al. Infigratinib Mediates Vascular Normalization, Impairs Metastasis, and Improves Chemotherapy in Hepatocellular Carcinoma. Hepatology. United States; 2019;69:943-58.

13. Weiss A, Adler F, Buhles A, Stamm C, Fairhurst RA, Kiffe M, et al. FGF401, A First-In-Class Highly Selective and Potent FGFR4 Inhibitor for the Treatment of FGF19-Driven Hepatocellular Cancer. Mol Cancer Ther. United States; 2019;18:2194-206.

14. Huynh H, Prawira A, Le TBU, Vu TC, Hao H-X, Huang A, et al. FGF401 and vinorelbine synergistically mediate antitumor activity and vascular normalization in FGF19-dependent hepatocellular carcinoma. Exp Mol Med. United States; 2020;52:1857-68.

15. Chan SL, Yen C-J, Schuler M, Lin C-C, Choo SP, Weiss K-H, et al. Abstract CT106: Ph I/II study of FGF401 in adult pts with HCC or solid tumors characterized by FGFR4/KLB expression. Cancer Res [Internet]. 2017;77:CT106 LP-CT106. Available from: http://cancerres.aacrjournals.org/content/77/13_Supplement/CT106.abstract

16. Guide for the Care and Use of Laboratory Animals, 8th edition. Washington (DC); 2011.

17. Huynh H, Ngo VC, Koong HN, Poon D, Choo SP, Toh HC, et al. AZD6244 enhances the anti-tumor activity of sorafenib in ectopic and orthotopic models of human hepatocellular carcinoma (HCC). J Hepatol. Netherlands; 2010;52:79-87. 
18. Casanovas O, Hicklin DJ, Bergers G, Hanahan D. Drug resistance by evasion of antiangiogenic targeting of VEGF signaling in late-stage pancreatic islet tumors. Cancer Cell. United States; 2005;8:299-309.

19. Yoshiji H, Kuriyama S, Yoshii J, Ikenaka Y, Noguchi R, Hicklin DJ, et al. Synergistic effect of basic fibroblast growth factor and vascular endothelial growth factor in murine hepatocellular carcinoma. Hepatology. United States; 2002;35:834-42.

20. Leach DR, Krummel MF, Allison JP. Enhancement of antitumor immunity by CTLA-4 blockade. Science. United States; 1996;271:1734-6.

21. Tian L, Goldstein A, Wang H, Ching Lo H, Sun Kim I, Welte T, et al. Mutual regulation of tumour vessel normalization and immunostimulatory reprogramming. Nature. England; 2017;544:250-4.

22. Khan KA, Kerbel RS. Improving immunotherapy outcomes with anti-angiogenic treatments and vice versa. Nat Rev Clin Oncol. England; 2018;15:310-24.

23. Kurebayashi $Y$, Ojima $H$, Tsujikawa $H$, Kubota N, Maehara J, Abe $Y$, et al. Landscape of immune microenvironment in hepatocellular carcinoma and its additional impact on histological and molecular classification. Hepatology. United States; 2018;68:1025-41.

24. Herbst RS, Soria J-C, Kowanetz M, Fine GD, Hamid O, Gordon MS, et al. Predictive correlates of response to the anti-PD-L1 antibody MPDL3280A in cancer patients. Nature. 2014;515:563-7.

25. Tumeh PC, Harview CL, Yearley JH, Shintaku IP, Taylor EJM, Robert L, et al. PD-1 blockade induces responses by inhibiting adaptive immune resistance. Nature. 2014;515:568-71.

26. Chen P-L, Roh W, Reuben A, Cooper ZA, Spencer CN, Prieto PA, et al. Analysis of Immune Signatures in Longitudinal Tumor Samples Yields Insight into Biomarkers of Response and Mechanisms of Resistance to Immune Checkpoint Blockade. Cancer Discov. 2016;6:827-37.

27. Nogova L, Sequist L V, Perez Garcia JM, Andre F, Delord J-P, Hidalgo M, et al. Evaluation of BGJ398, a Fibroblast Growth Factor Receptor 1-3 Kinase Inhibitor, in Patients With Advanced Solid Tumors Harboring Genetic Alterations in Fibroblast Growth Factor Receptors: Results of a Global Phase I, Dose-Escalation and Dose-Expansion St. J Clin Oncol Off J Am Soc Clin Oncol. 2017;35:157-65.

28. Dawkins J, Webster RM. The hepatocellular carcinoma market. Nat Rev Drug Discov. England; 2019;18:13-4.

29. Tallarida RJ. Drug synergism: its detection and applications. J Pharmacol Exp Ther. United States; 2001;298:865-72.

\section{Figures}



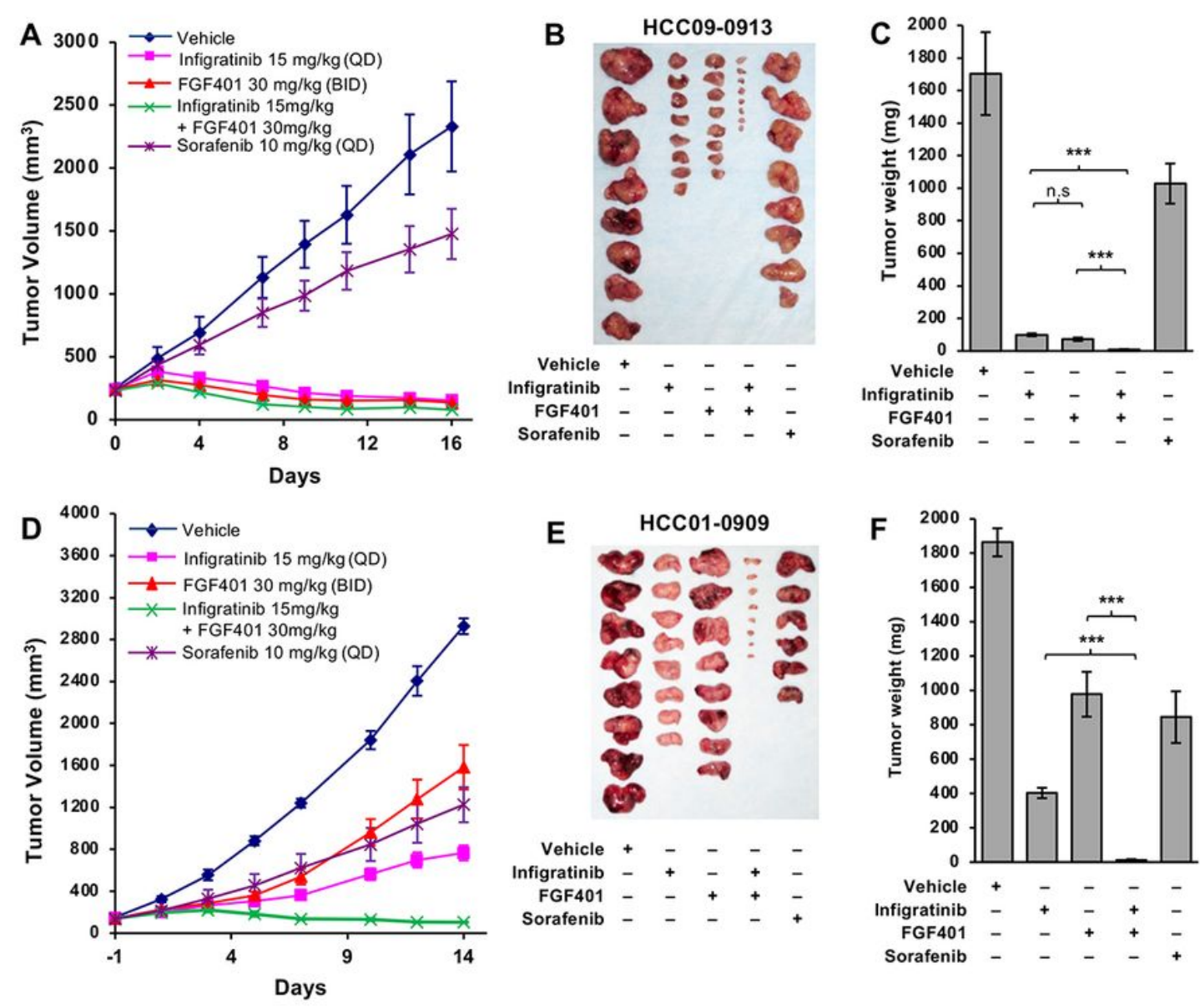

Figure 1

\section{Figure 1}

The effects of infigratinib, FGF401, sorafenib, and infigratinib/FGF401 on tumor growth. High FGF19expressing HCC09-0913 and high FGFR2/3-expressing HCC01-0909 tumors were subcutaneously implanted and mice ( $n=10$ per group) were subsequently treated with: 1) $200 \mu$ l vehicle (control), 2) 15 $\mathrm{mg} / \mathrm{kg}$ infigratinib once daily, 3) $30 \mathrm{mg} / \mathrm{kg}$ FGF401 twice daily, 4) combined infigratinib and FGF401, or 5) $10 \mathrm{mg} / \mathrm{kg}$ sorafenib once daily. Treatments were initiated when the tumors reached approximately 170$250 \mathrm{~mm} 3$. Mean tumor volume over time $(A, D)$, tumor size $(B, E)$, and median tumor weights $(C, F)$ at sacrifice are shown. ${ }^{*} p<0.05 ; * \star p<0.01 ; * \star \star p<0.001 ;$ n.s. not significant. 

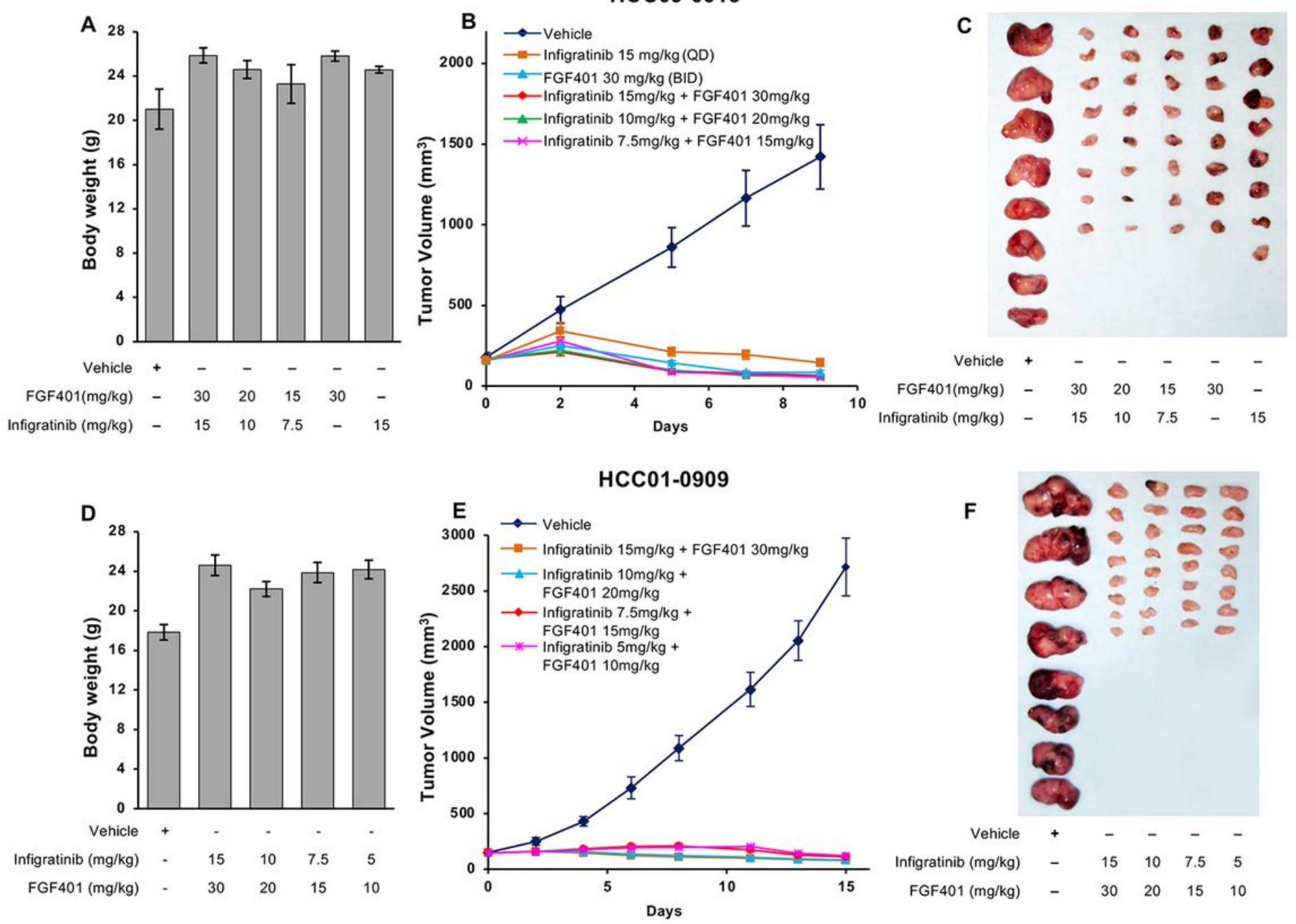

Figure 2

\section{Figure 2}

Dose-dependent effects of infigratinib/FGF401 on tumor growth. Mice bearing high FGF19- and FGFR2/3expressing HCC09-0913 xenografts (eight mice per group) were administered vehicle, $15 \mathrm{mg} / \mathrm{kg}$ infigratinib, $30 \mathrm{mg} / \mathrm{kg}$ FGF401, and three combinations (15:30, 10:20, and 7.5:15 infigratinib:FGF401) as described in Figure 1 for 9 days. Mice bearing high FGFR2/3-expressing but low FGF19 HCC01-0909 xenografts (eight mice per group) were administered vehicle and four combinations (15:30, 10:20, 7.5:15, and 5:10 infigratinib: FGF401) for 15 days. Infigratinib once daily and FGF401 twice daily were administered orally. Treatment was initiated when the tumors reached sizes of approximately 170-250 mm3. Tumor growth was monitored, and tumor volume was calculated as described previously [12]. Body weight at sacrifice $(A, D)$, tumor volume over time $(B, E)$, and tumor size at sacrifice $(C, F)$ are shown. 

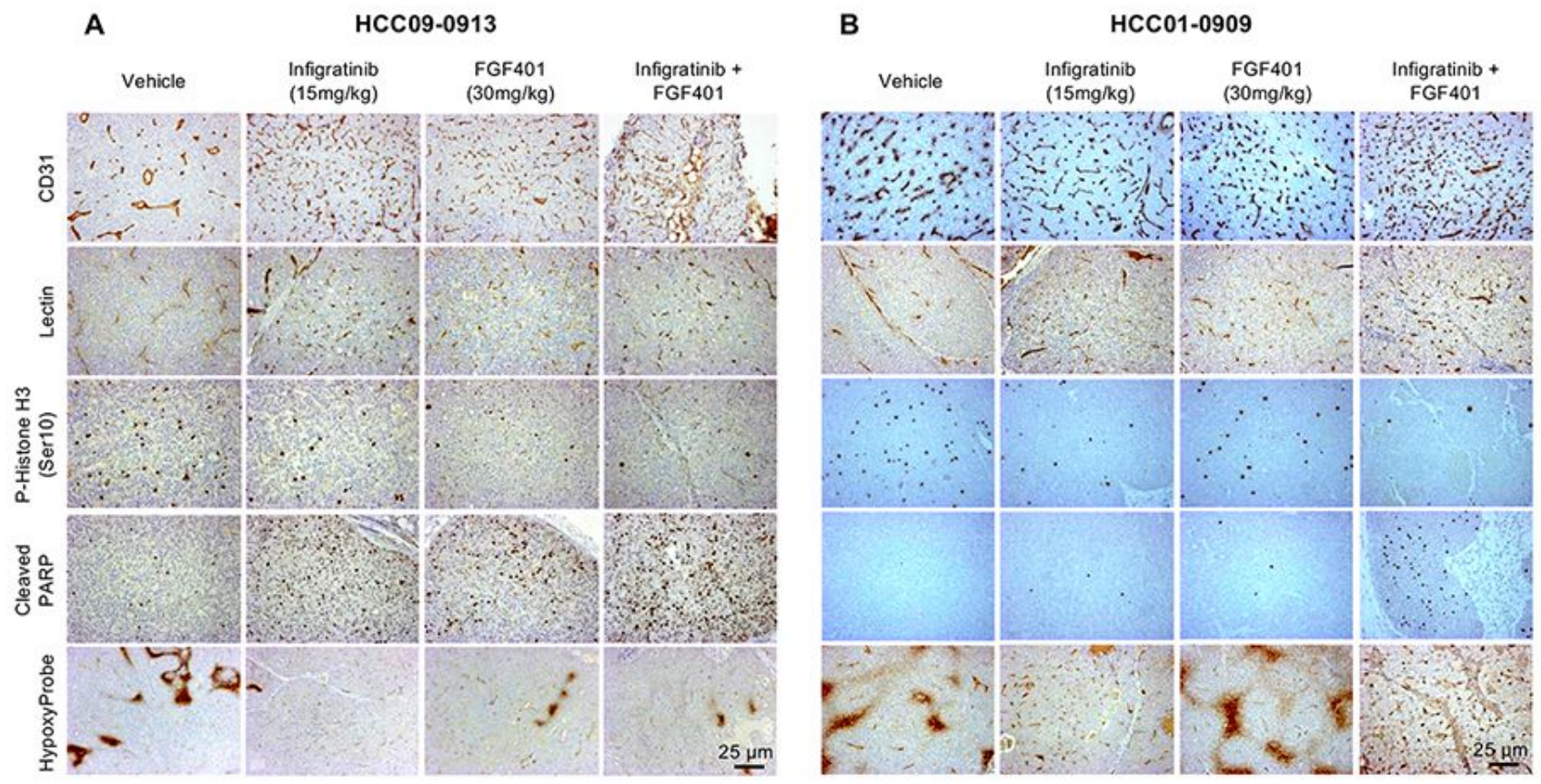

Figure 3

\section{Figure 3}

The effects of infigratinib/FGF401 on vascular normalization, cell proliferation, apoptosis, and hypoxia in HCC models. Mice bearing HCC09-0913 and HCC01-0909 xenografts were treated with vehicle, infigratinib, FGF401, or infigratinib/FGF401 for 16 days as described in Figure 1, and subsequently subjected to lectin perfusion followed by pimonidazole $\mathrm{HCl}$ injection. Tumors were collected $2 \mathrm{~h}$ after the last treatments and were processed for immunohistochemistry. Representative images of tumor sections stained for p-Histone H3 Ser10, cleaved PARP, CD31, lectin, and Hypoxyprobe to assess cell proliferation, apoptosis, microvessels, productive blood vessels, and tumor hypoxia, respectively as described [12]. Bars: $25 \mu \mathrm{m}$. 

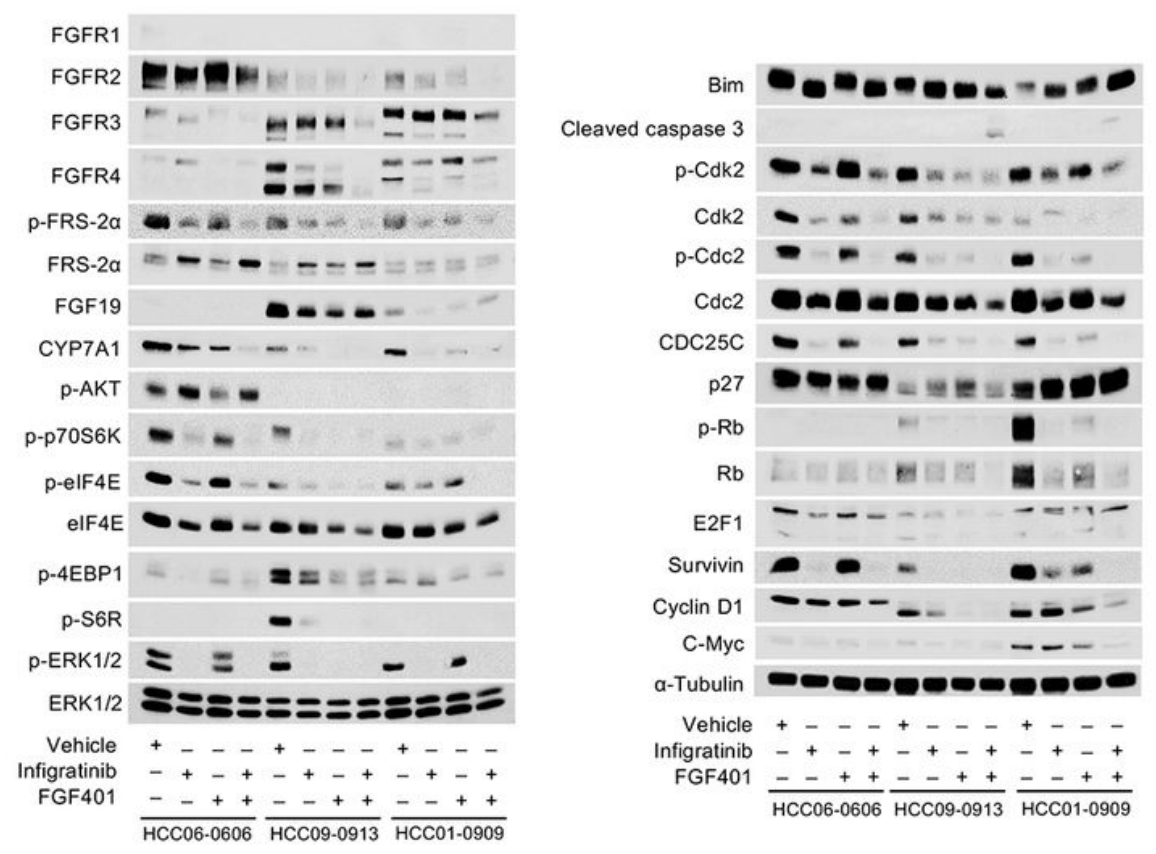

\section{Figure 4}

\section{Figure 4}

The effects of infigratinib/FGF401 on the pathways involved in cell growth, invasion, metastasis, and survival. Mice bearing indicated tumors ( $n=3$ per group) were treated with 1$)$ vehicle, 2 ) infigratinib, 3 ) FGF401, and 4) infigratinib/FGF401 for 4 days. Tumors were collected $2 \mathrm{~h}$ after the last dose of drugs and lysates were subjected to Western blot analysis as described [12]. Representative blots are shown. 

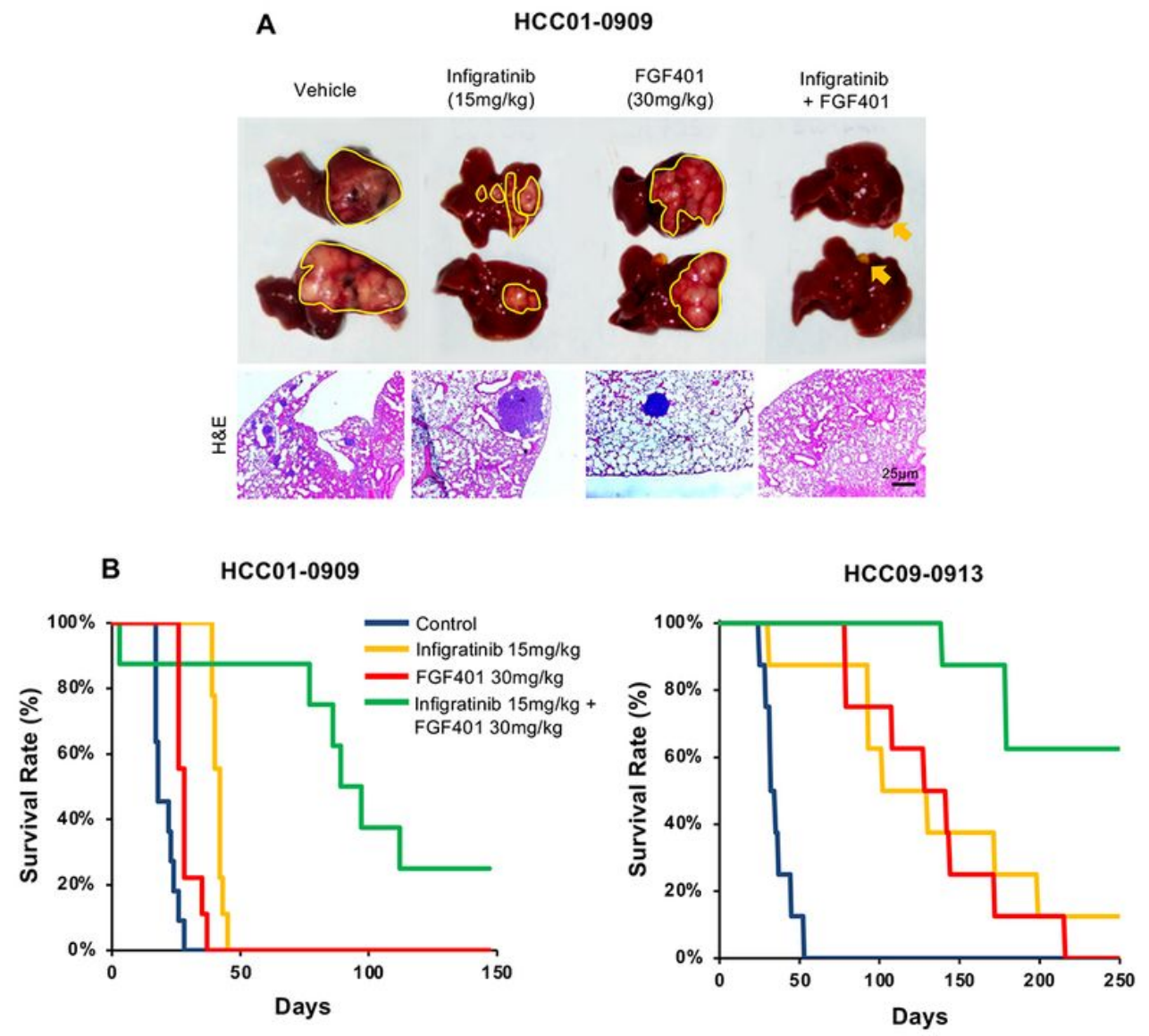

Figure 5

\section{Figure 5}

The effects of infigratinib/FGF401 on tumor growth and the survival of mice bearing orthotopic HCC. Mice bearing HCC01-0909 and HCC09-0913 tumors ( $\mathrm{n}=10$ per group) were treated with 1) vehicle, 2 ) infigratinib, 3) FGF401, or 4) infigratinib/FGF401 for 28 days. Treatments started when the tumors reached approximately 100-150 mm3. The sizes of the primary orthotopic HCC01-0909 tumors and representative photographs of lung sections with metastatic nodules $(A)$ and representative KaplanMeier survival analyses for HCC01-0909 and HCC90-0913 (B) are shown. Bars: $25 \mu \mathrm{m}$. 


\section{HCC01-0909 (Humanized mice)}
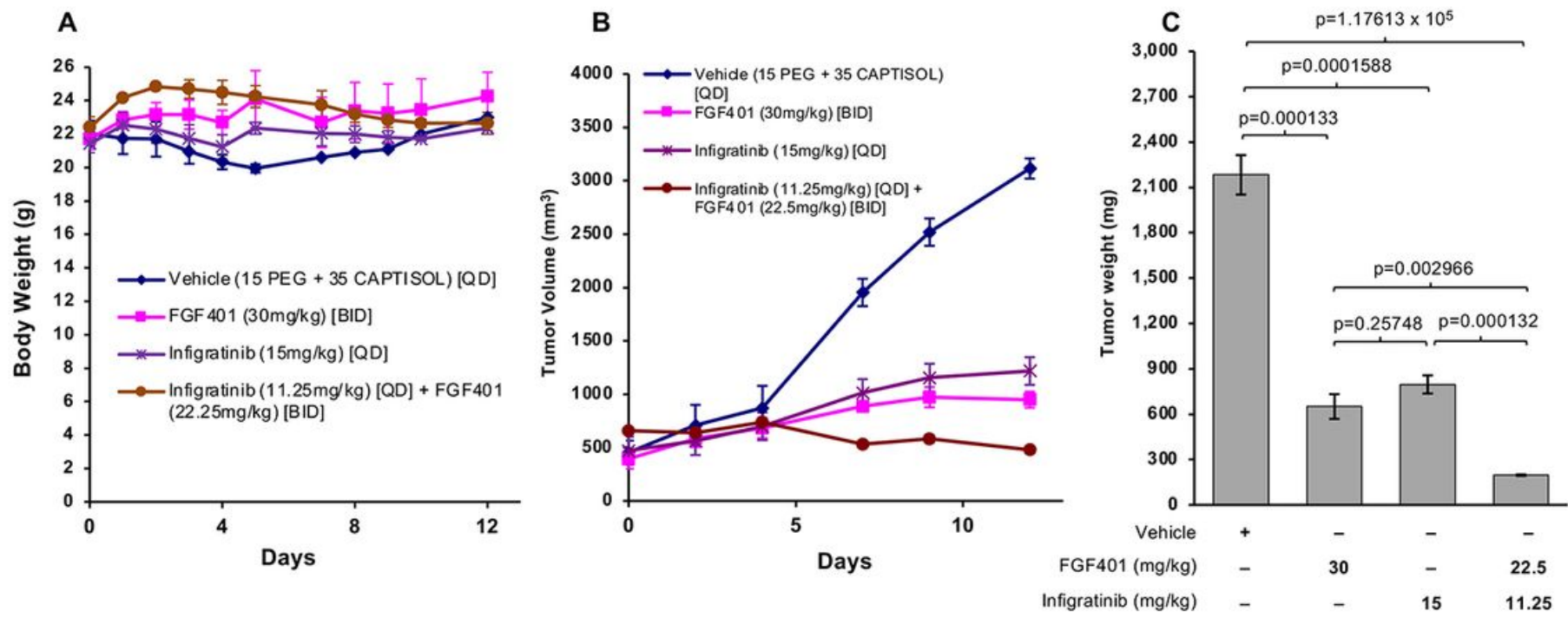

Figure 6

\section{Figure 6}

Infigratinib/FGF401 induces tumor regression in humanized mouse model. HCC01-0909 tumor was subcutaneously implanted in CD34+ hu-NSG humanized mice. They were randomized into 4 groups and treated with 1) $200 \mu \mathrm{l}$ vehicle (control), 2) $15 \mathrm{mg} / \mathrm{kg}$ infigratinib once daily, 3) $30 \mathrm{mg} / \mathrm{kg}$ FGF401 twice daily, 4) combined $11.25 \mathrm{mg} / \mathrm{kg}$ infigratinib and $22.5 \mathrm{mg} / \mathrm{kg}$ FGF401 for 11 days. Body weight over time $(A)$, tumor volumes over time $(B)$, and tumor weight at sacrifice $(C)$ of the drug-treated and vehicle-treated tumors at sacrifice are shown. Differences in tumor weight were compared using one-way ANOVA followed by the Tukey-Kramer HSD post-hoc test. $p<0.05$ is considered to be significant. SE, standard error of the mean.

\section{Supplementary Files}

This is a list of supplementary files associated with this preprint. Click to download.

- SupplementaryFigures.pdf 
- SupplementaryData.docx

Page 20/20 